Article

\title{
Comparison of Whole-Cell SELEX Methods for the Identification of Staphylococcus Aureus-Specific DNA Aptamers
}

\author{
Jihea Moon, Giyoung Kim *, Saet Byeol Park, Jongguk Lim and Changyeun Mo \\ National Academy of Agricultural Science, 310 Nongsaengmyeng-ro, Wansan-gu, Jeonju 560500, \\ Korea; E-Mails: mmir95@gmail.com (J.M.); veryvenus0911@gmail.com (S.B.P.); \\ limjg@korea.kr (J.L.); cymoh100@korea.kr (C.M.) \\ * Author to whom correspondence should be addressed; E-Mail: giyoung@korea.kr; \\ Tel.: +82-63-238-4111; Fax: +82-63-238-4105.
}

Academic Editor: Stephane Evoy

Received: 6 February 2015 / Accepted: 9 April 2015 / Published: 15 April 2015

\begin{abstract}
Whole-cell Systemic Evolution of Ligands by Exponential enrichment (SELEX) is the process by which aptamers specific to target cells are developed. Aptamers selected by whole-cell SELEX have high affinity and specificity for bacterial surface molecules and live bacterial targets. To identify DNA aptamers specific to Staphylococcus aureus, we applied our rapid whole-cell SELEX method to a single-stranded ssDNA library. To improve the specificity and selectivity of the aptamers, we designed, selected, and developed two categories of aptamers that were selected by two kinds of whole-cell SELEX, by mixing and combining FACS analysis and a counter-SELEX process. Using this approach, we have developed a biosensor system that employs a high affinity aptamer for detection of target bacteria. FAM-labeled aptamer sequences with high binding to $S$. aureus, as determined by fluorescence spectroscopic analysis, were identified, and aptamer A14, selected by the basic whole-cell SELEX using a once-off FACS analysis, and which had a high binding affinity and specificity, was chosen. The binding assay was evaluated using FACS analysis. Our study demonstrated the development of a set of whole-cell SELEX derived aptamers specific to S. aureus; this approach can be used in the identification of other bacteria.
\end{abstract}

Keywords: aptamer; biosensor; pathogen detection; whole-cell SELEX 


\section{Introduction}

Enterotoxin-producing Staphylococcus aureus (S. aureus) cause nausea, stomach cramps, vomiting, and diarrhea [1]. In some cases, such as in infants, elderly or immunocompromised individuals, S. aureus infections can be severe [2]. S. aureus outbreaks have been associated with meat, poultry, and their products; however, $S$. aureus is also present in the air, in dust, water, food, humans, and animals [3]. In fact, $S$. aureus is one of the most common and important foodborne bacteria in the world [4]. In Korea, S. aureus caused 6.8\% of foodborne illnesses recorded during 2002-2012 [5]. In the USA, 241,188 illnesses, 1064 hospitalizations, and six deaths due to staphylococcal food poisoning are reported annually [6].

Recently, the control of $S$. aureus outbreaks has gained social and economic importance globally [3] emphasizing the need for a rapid method of detecting $S$. aureus. Although methods for detection and control of $S$. aureus are well established, conventional methods, such as the standard plate count method after selective enrichment in broth, are lengthy, complicated and require multiple steps $[4,7]$. To overcome these factors, biosensors that allow rapid and efficient detection of foodborne bacteria have been developed [8,9]. Biosensors are composed of a bioreceptor, transducer, and a data recording and display device. In this context, a bioreceptor is a biological molecular species, such as an antibody, enzyme, protein, or nucleic acid that utilizes a biochemical mechanism for the recognition of a biological molecule [10]. Antibodies have commonly been used as bioreceptors that exhibit high affinity and a specific binding capability between antigen and antibody. However, the use of antibodies has notable weaknesses, such as requiring animal hosts, although they are not well tolerated, as well as a very expensive and laborious production process, batch-to-batch variation, sensitivity to temperature, irreversible denaturation, and limited shelf life [11].

Consequently, aptamers have been developed that can be used instead of antibodies. Aptamers are composed of ssDNA or RNA, and exhibit highly selective and specific binding affinities for target molecules [12]. Aptamers, shorter than 40 nucleotides, are produced with great accuracy and reproducibility, by chemical synthesis, and are stable across temperatures and during long-term storage [13]. Aptamers are identified by means of Systematic Evolution of Ligands by EXponetial enrichment (SELEX). SELEX is a technique by which random libraries of oligonucleotides can be screened by in vitro selection using targets and can then be amplified by PCR [12]. Among SELEX technologies, whole-cell SELEX is a method developed and modified for creating aptamers bound to live bacteria [14]. Whole-cell SELEX comprises multiple steps: (1) screening of random nucleic acid bound to target bacteria, (2) repeated separation and exponential amplification of the oligonucleotide, and (3) cloning and sequencing of the specific binding molecules ultimately identified [15]. Because the target in whole-cell SELEX is live pathogenic bacteria, aptamers can find and bind more efficiently to the surface molecules of live bacteria than those in other SELEX approaches [16]. Therefore, the aptamers derived in whole-cell SELEX may have little cross-reactivity to non-target bacteria [17].

Despite of the advantages of whole-cell SELEX, its success rate is only below $50 \%$ as to the complex structure of target bacteria [18]. Therefore, to improve development of successful aptamers, various SELEX processes have emerged, designed to suit the requirements of specific individual purposes [19]. In this study, we designed, developed, and characterized DNA aptamers specific to $S$. aureus, using two kinds of modified whole-cell SELEX approaches for improving the specificity and selectivity of 
the aptamers. Because flow cytometry is a crucial method for selecting target aptamers specifically bound to cells [20], multiple FACS sorting steps were incorporated in the modified SELEX process to further improve the specificity. We then compared their binding activity using FACS analysis.

\section{Materials and Methods}

\subsection{Biological and Chemical Materials}

Salmonella typhimurium (KCCM 12041), Salmonella enteritidis (KCCM 12021), Escherichia coli (KCCM 11234), and Staphylococcus aureus (KCCM 12103) were obtained from the Korean Collection of Type Cultures (Daejun, Korea). Tryptic soy (TS) agar, BBL eosin methylene blue (EMB) agar, XLT4 agar base, XLT4 agar supplement, Baird-Parker agar base, and EY tellurite enrichment were purchased from BD Difco (Sparks, MD, USA). The initial ssDNA library and the primers used for amplification were synthesized and purified by polyacrylamide gel electrophoresis (PAGE; Bioneer Co., Ltd, Daejeon, Korea). Phosphate-buffered saline (PBS, pH 7.4) was purchased from Sigma (St. Louis, MO, USA). PCR tubes, reagents, and polymerase were obtained from Takara (Shiga, Japan). LE agarose and TAE buffer were purchased from Lonza (Rockland, ME, USA). The Qiagen MinElute gel extraction kit was obtained from Qiagen (Hilden, Germany). The In-Fusion HD Cloning kit was purchased from Clontech (Mountain View, CA, USA).

\subsection{Bacterial Strains}

Stock cultures of $S$. aureus, S. typhimurium, S. enteritidis, and E. coli were grown overnight at $35^{\circ} \mathrm{C}$ in brain-heart infusion medium (Difco, Franklin Lakes, NJ, USA). Cells were harvested by centrifugation, washed three times in PBS, and finally suspended in $100 \mu \mathrm{L}$ of PBS prior to use in experiments.

\subsection{Preparation of DNA Library}

The DNA template was synthesized as ssDNA containing 40 random nucleotides, viz. 5'-CGGATGCGAATTCCCTAATACGACTCACTATAGGGCGT-N 40 -GGTGGATCCATATTCCT ACTCG-3'. The initial DNA library was prepared by PCR amplification with Pyrobest DNA polymerase (Takara).

\subsection{PCR Optimization}

PCR was performed prior to binding of the SELEX library to $S$. aureus to optimize the annealing temperature. One hundred $\mu \mathrm{L}$ PCR mix consisting of $1 \mathrm{ng}$ of either capture of reporter SELEX DNA templates, 25 pmol of appropriate primer (unlabeled or FAM[fluorescein]-labeled 20mer primers), $10 \mu \mathrm{L}$ of $10 \times$ Pyrobest buffer, $10 \mathrm{mM}$ dNTP (Takara), 2 units of Pyrobest DNA polymerase, and double distilled water ( $\mathrm{ddH}_{2} \mathrm{O}$; Biosesang, Gyeonggi-do, Korea) was used for each PCR in a thermal cycler (BioRad, Hercules, CA, USA). PCR annealing temperature was studied between $56{ }^{\circ} \mathrm{C}$ and $70{ }^{\circ} \mathrm{C}$ (data not shown). Optimal PCR conditions were determined to be an initial denaturation at $98{ }^{\circ} \mathrm{C}$ for 
$2 \mathrm{~min}$, followed by 30 cycles, each consisting of $98^{\circ} \mathrm{C}$ for $30 \mathrm{~s}, 67^{\circ} \mathrm{C}$ for $15 \mathrm{~s}, 72{ }^{\circ} \mathrm{C}$ for $30 \mathrm{~s}$, and a final extension at $72{ }^{\circ} \mathrm{C}$ for $5 \mathrm{~min}$.

\subsection{SELEX Procedures}

The whole-cell SELEX process is outlined in Figure 1. To improve the selectivity of the aptamer, SELEX was designed using two types of processes. A total of 10 rounds of SELEX and 6 rounds of counter-SELEX were performed. In case of Figure 1a, the sorting step using flow cytometry, was conducted after all the SELEX stages had been completed. In the case described in Figure 1b, the aptamer candidates for binding to $S$. aureus were sorted using flow cytometry after the third, sixth, and tenth rounds of SELEX or counter-SELEX. SELEX was performed using a modification of Moon's methods [21].

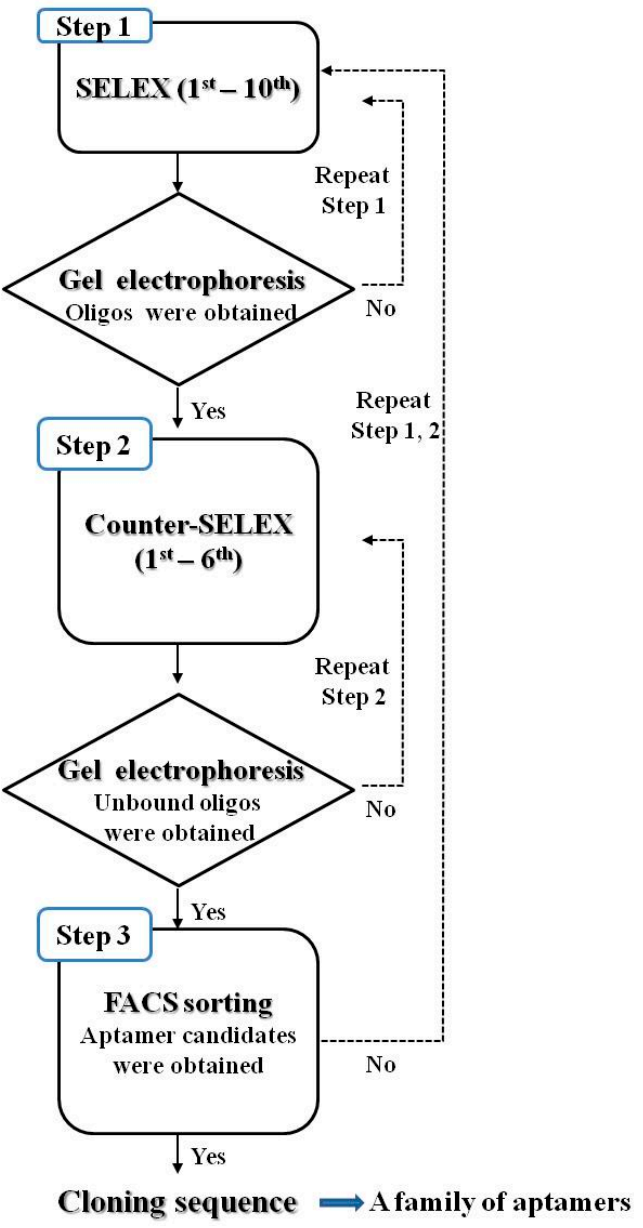

(a)

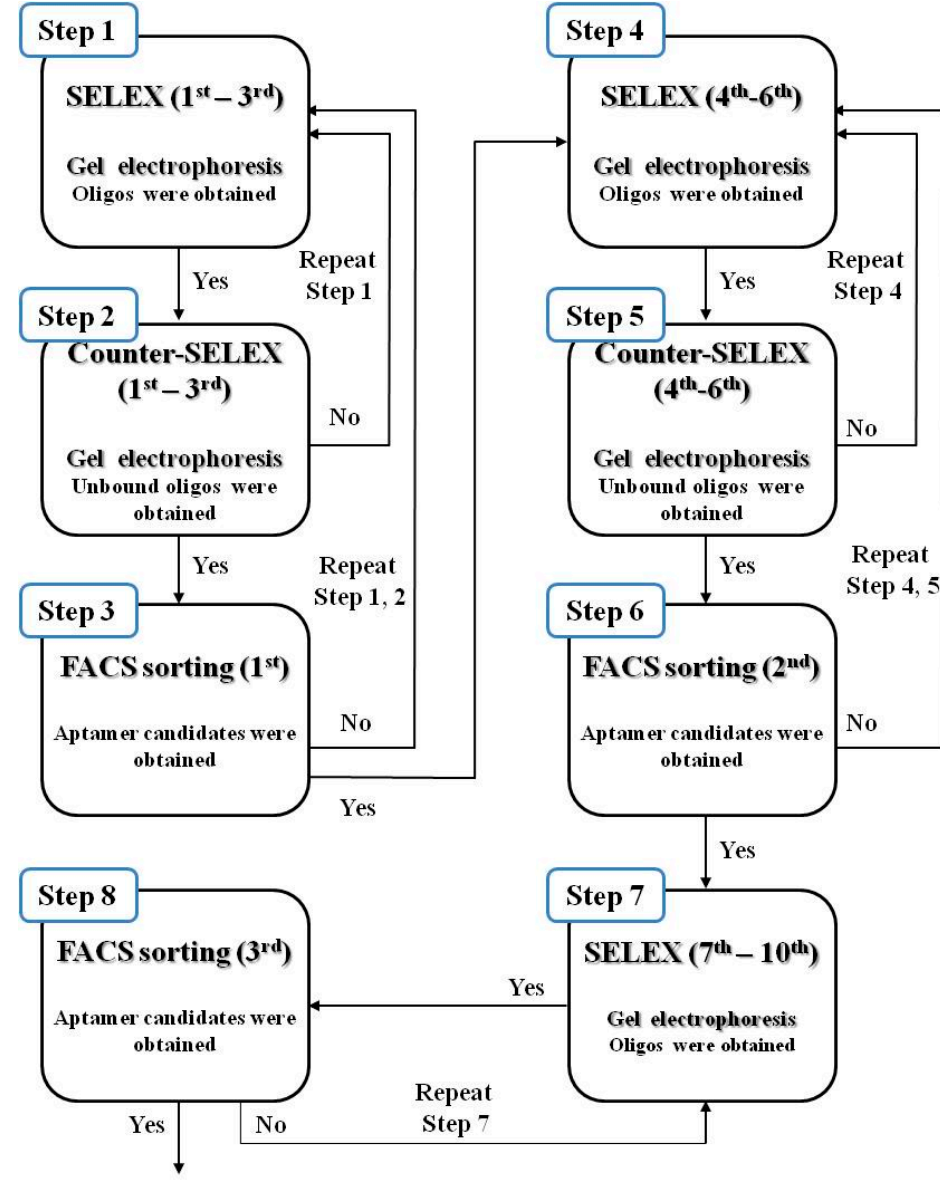

Cloning sequence $\longrightarrow B$ family of aptamers

(b)

Figure 1. (a) Schematic diagram of the basic whole-cell SELEX procedures used to isolate DNA aptamers with high binding affinity for Staphylococcus aureus; (b) Schematic diagram of the modified whole-cell SELEX process used to isolate DNA aptamers with a high binding affinity for Staphylococcus aureus.

In the selection rounds, $1.0 \mu \mathrm{g}$ of double-stranded DNA library, dissolved in $50 \mu \mathrm{L}$ of PBS ( $\mathrm{pH} 7.4$, Sigma, St. Louis, MO, USA) was denatured by heating at $95^{\circ} \mathrm{C}$ for $10 \mathrm{~min}$ and then cooling 
on ice for $10 \mathrm{~min}$. The denatured ssDNA was incubated with $100 \mu \mathrm{L}$ of $1.0 \times 10^{8} \mathrm{cfu} / \mathrm{mL}$ S. aureus, with shaking $(1500 \mathrm{rpm})$ at room temperature for $45 \mathrm{~min}$. Unbound ssDNA was removed by centrifugation at $8000 \mathrm{~g}$ for $10 \mathrm{~min}$. Then, the bound ssDNA was eluted in $500 \mu \mathrm{L}$ of double distilled water $\left(\mathrm{ddH}_{2} \mathrm{O}\right)$ by heating bacteria-DNA complexes at $95{ }^{\circ} \mathrm{C}$ for $10 \mathrm{~min}$. After centrifugation, the supernatant was used as the aptamer candidate template for amplification by PCR. Electrophoresis on a $2.0 \%$ agarose gel was used to confirm the purity and to verify the size of the PCR products. Some of the PCR products, insufficiently amplified to allow detection of the band size on the Gel-doc system (BioRad), were purified by Ultracel (Milipore, Billerca, MA, USA). After verification of the band on a gel, the target materials were carefully excised and extracted using a MinElute gel extraction kit (Qiagen, Hilden, Germany). These extraction methods were also used in the rounds of counter-selection. After incubation with non-target bacteria, bound ssDNA was removed by centrifugation, while the unbound aptamer candidates in the supernatant were collected for one more round of counter-SELEX or SELEX. In case of PCR failure caused by human errors or unknown reasons, aptamer candidates selected from the previous step were used for the remaining process.

\subsection{Fluorescence-Activated Cell Sorting (FACS)}

Cell sorting was performed after the third, sixth, and tenth rounds of counter-SELEX or SELEX. For cell sorting, the selected DNA was fluorescently labeled via PCR amplification with 5'-FAM- and 3'-FAM-modified primers. The FAM-dsDNA was denatured to ssDNA by heat treatment. Binding assays involved incubating $1.0 \mu \mathrm{g}$ of FAM-labeled ssDNA with $10^{8}$ cells of $S$. aureus in PBS for $45 \mathrm{~min}$ and then washing twice with PBS. Cells were resuspended in $100 \mu \mathrm{L}$ of PBS for flow cytometric analysis. Forward scatter, side scatter, and fluorescence intensity were measured and gated fluorescence intensity above background level was quantified. Aptamer candidates bound to $S$. aureus were sorted by FACS and then amplified with non-labeled primers. These amplified aptamer candidates were cloned and sequenced at Macrogen (Seoul, Korea). The aptamer sequences were analyzed using a commercial genetic analysis program (CLC Workbench 6, Aarhus, Denmark).

\subsection{Characterization of Binding Parameters of Aptamers to Bacteria}

To test the binding affinity of aptamer candidates, fluorescence analysis was performed using an Infinite M1000 spectrophotometer (TECAN, Männedorf, Switzerland). The binding affinity of aptamer candidates was determined by incubating $S$. aureus cells $\left(10^{8}\right)$ with varying concentrations of FAM-labeled aptamer candidates in $500 \mu \mathrm{L}$ of PBS for $45 \mathrm{~min}$ at room temperature. Cells were then washed twice with $500 \mu \mathrm{L}$ of PBS, suspended in $100 \mu \mathrm{L}$ of PBS, and then analyzed. The FAM-labeled unselected ssDNA library was used as a negative control for non-specific binding. All the binding assay experiments were repeated 3 times. The mean fluorescence intensity of target bacteria labeled with aptamer candidates was used to calculate the specific binding by subtracting the mean fluorescence intensity of nonspecific binding produced by unselected library DNA. The equilibrium dissociation constants $\left(K_{d}\right)$ of the fluorescent ligands were obtained by fitting the dependence of the fluorescence intensity of specific binding on the concentration of the ligands to the equation $Y=B_{\max } X /\left(K_{d}+X\right)$ using Graphpad Prism 6 (GraphPad Software, CA, USA). 


\subsection{Aptamer-Binding Assays and Prediction of Candidate Aptamer Sequences}

Aptamer A14 was evaluated for binding activity and cross reactivity with related and unrelated pathogenic bacteria. The FAM-labeled aptamer $(1.0 \mathrm{nmol})$ was dissolved in $50 \mu \mathrm{L}$ of $\mathrm{ddH}_{2} \mathrm{O}$, and denatured by heating $\left(95{ }^{\circ} \mathrm{C}\right.$ for $10 \mathrm{~min}$ ) and cooling (on ice for $10 \mathrm{~min}$ ). Then, $1.0 \mathrm{nmol}$ of FAM-labeled aptamer was incubated with target bacteria $\left(10^{8} \mathrm{CFU}\right)$ in $500 \mu \mathrm{L}$ of PBS for 45 min at room temperature. After incubation, samples were centrifuged at $8000 \times \mathrm{g}$ for $10 \mathrm{~min}$ and the supernatant removed. Five hundred microliters of PBS was added to the samples, which were then mixed well. After one more PBS wash step, $50 \mu \mathrm{L}$ of PBS was added for the binding assay. The fluorescently labeled analytes were monitored at 505-535 nm, using an Infinite M1000 microplate reader (TECAN) at an excitation of $494 \mathrm{~nm}$. To evaluate cross-reactivity, the binding assays were repeated using E. coli, S. typhimurium, and S. enteritidis. For further characterization of binding affinity, different concentrations of FAM-aptamers $(0.1,0.3,0.5,0.7$, and $3 \mathrm{nmol})$ were used and analyzed by FACS. FACS analysis was performed on 20,000 cells. The secondary structure of aptamers binding to $S$. aureus was predicted using the genetic analysis program (CLC Workbench 6).

\section{Results and Discussion}

\subsection{Screening of Aptamer Candidates in the Basic Whole-Cell SELEX}

A total of 10 rounds of SELEX and 6 rounds of counter-SELEX were performed to select aptamers with selectivity for $S$. aureus. Cell-bound aptamers were separated by cell sorting using FACS. The selected aptamer candidates were cloned and sequenced (Figure 1a). From these, the majority of sequences could be classified into 24 families. These unique aptamer candidates were pre-screened for binding interaction with $S$. aureus and E. coli cells $\left(10^{7}\right)$ by using $1.0 \mathrm{nmol}$ of each sequence by fluorescence spectrometry (Figure 2a). A15 showed the highest fluorescence, followed by A14. Although comparatively lower affinity for $S$. aureus was shown by aptamer A2 and A20, they also showed lower affinity to $E$. coli. The sequences selected from the randomized region of A2, A14, A15 and A20 are in Table 1. Error bars on each graph indicate the standard deviation of fluorescence signals from three samples.

Table 1. Sequences of selected aptamers for Staphylococcus aureus.

\begin{tabular}{cc}
\hline Aptamers & Sequences \\
\hline A2 & ACGGGCGTGGGAGGCAATGCCTTGCTTGTAGGCTTCCCCTGTGCGCG \\
A14 & CACACCGCAGCAGTGGGAACGTTTCAGCCATGCAAGCATCACGCCCGT \\
A15 & CACGCGCAAACAGATTAACACTCCGCCTAAGTCTGCCGCACGC \\
A20 & GCGTGCAGCGGGGGCTGCGCGGTGGAGTGCTGTGGGCG \\
B3 & GCGTGCGGAGCCAGGATGGGAGGTCTGTAGGTCTGCGGGGCGTG \\
B6 & GCGTGTCGGTGTCTGCCGGGGGATGTGGAGGCTGGGTGTTGCGCG \\
B7 & GCGTGGGCGGGCTACCTGGCTAGTACGCCATGATGCCTGCACGCG \\
B15 & CACGCGCAAACAGATTAACACTCCGCCTAAGTCTGCCGCACGC \\
\hline
\end{tabular}




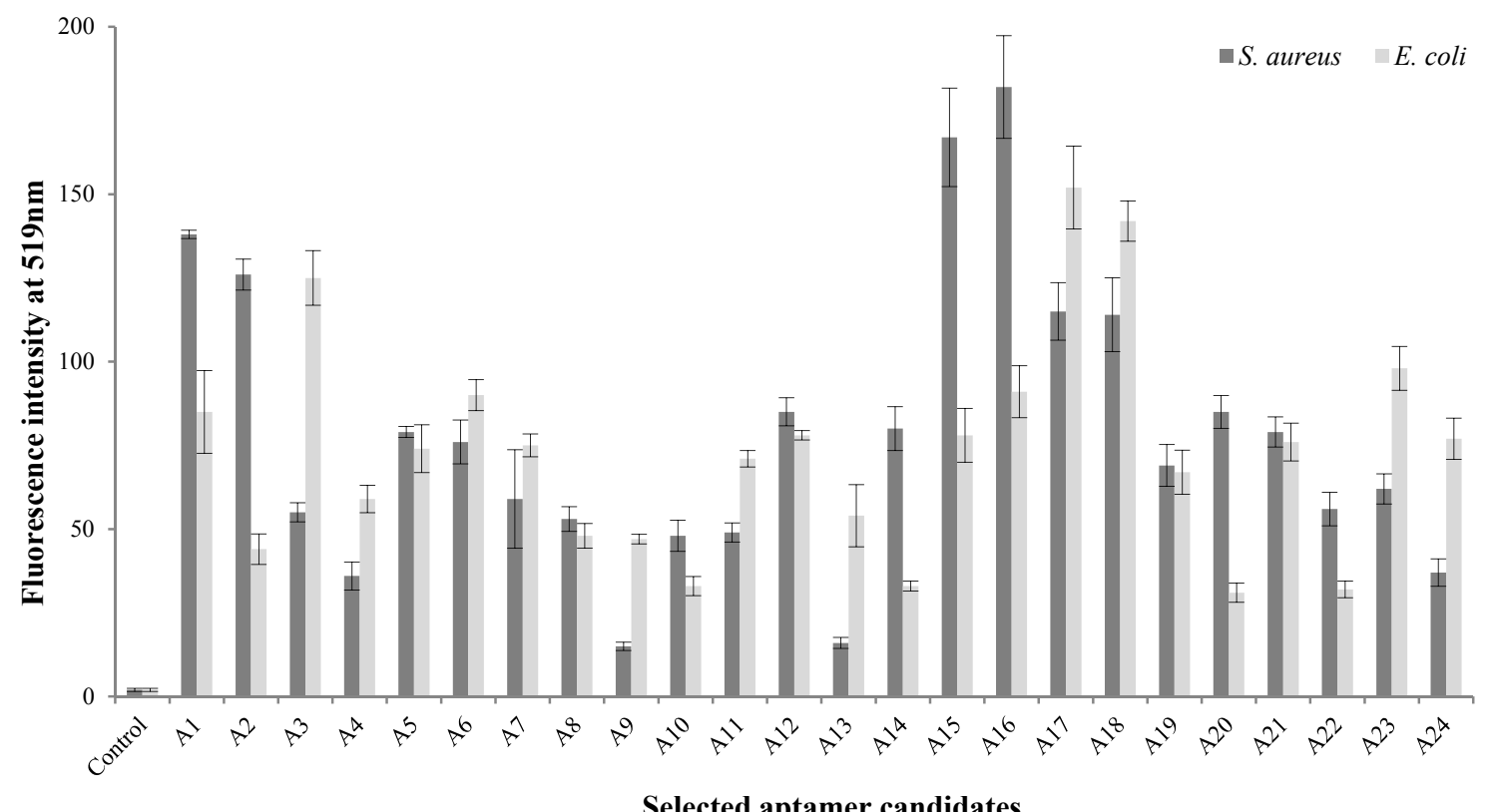

(a)

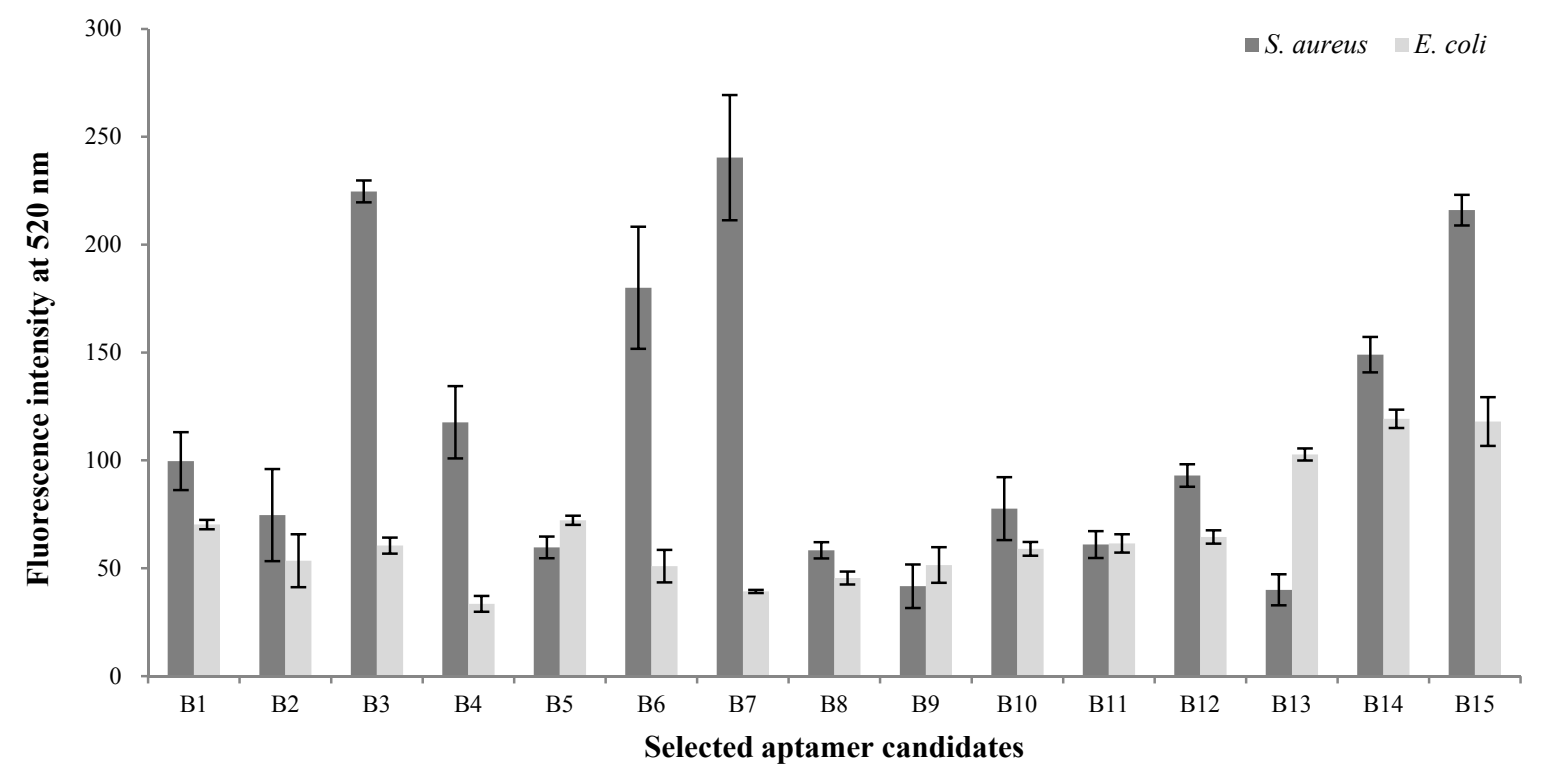

(b)

Figure 2. (a) Validation of the binding specificity of selected aptamer candidates by using the basic whole-cell SELEX procedure; (b) Validation of binding specificity of selected aptamer candidates by using the modified whole-cell SELEX procedure.

FACS analysis is an important method for developing aptamers specifically bound to target cells [22]. According to a previous study, nucleic acids (aptamer candidates), selected by whole-cell SELEX, would have good affinity for, but also poor specificity to target bacteria. Thus, FACS analysis could be a crucial step in improving their affinity and specificity. We compared the selective intensity and specificity of aptamers A2, A14, A15, and A20 (1.0 nmol each) for S. aureus and E. coli (10 ${ }^{8}$ cells each) by flow cytometry. The control groups were $S$. aureus, and $E$. coli to which no ssDNA had been added. Compared with the $S$. aureus control group, the selected aptamer candidates demonstrated good binding affinity. The selected aptamers A14 and A15, demonstrated stronger binding affinity for 
S. aureus than did A2 and A20 (Figure S1). However, A15 showed some binding affinity for E. coli as compared with the E. coli control group.

\subsection{Screening of Aptamer Candidates in the Modified Whole-Cell SELEX}

In the case of the modified whole-cell SELEX, the SELEX process was repeated with SELEX per se, cell sorting by FACS analysis, and counter-SELEX for improving the specificity and selectivity of aptamer. Our SELEX approach is summarized in Figure 1b. First, three rounds of SELEX and counter-SELEX were conducted for screening the aptamer candidates, specific to target bacteria. The first cell sorting was performed as described and sorted DNAs were amplified for the next step of the process [21]. Second, three rounds of counter-SELEX and SELEX were carried out after each other. After six rounds of SELEX, the second cell sorting step was performed. Third, three rounds of counter-SELEX and four rounds of SELEX were performed, and then the last cell sorting step was used for retaining the aptamer candidates. Using $S$. aureus as target cell and S. typhimurium and E. coli as negative control for counter-selection, we generated aptamers targeting molecules only present on the cell surface of $S$. aureus [16]. In addition, we added multiple cell sorting steps to obtain aptamers that are reliable in whole-cell SELEX. Therefore, our modified whole-cell SELEX process combined with cell sorting and counter-SELEX allowed us to optimize selectivity and specificity over the established SELEX method.

Moreover, in this study, the entire DNA library not bound to $S$. aureus was removed in the repeated selection and cell-sorting process. Observation of a target band on the agarose gel after the rounds of selection suggested that aptamer candidates could specifically bind to $S$. aureus. After the selection process, the sorted aptamer pools were cloned and sequenced, resulting in a total of 34 sequences. According to the sequencing results and the homology of the DNA sequences, the majority of the sequences could be classified into 15 families. These aptamer candidates were synthesized and modified with FAM, to allow determination of the binding affinity by fluorescent spectrophotometry. Spectrophotomeric analysis has recently been used to enhance the ease, rapidity, and sensitivity of the process $[20,21,23]$. The selected FAM-dsDNA aptamer candidates were denatured by heat treatment for transferring to FAM-ssDNA. Their sequences selected from the randomized region are shown in Table 1. They were bound to $S$. aureus, and were then analyzed and compared. To characterize the specificity of aptamer candidates, FAM-labeled aptamers were tested against E. coli. Aptamer B7 showed the highest florescence, followed by B3, B6, and B15, while comparatively lower affinity was shown by aptamer B9 and B13 (Figure 2b).

Aptamer B3, B6, B7, and B15 were analyzed by FACS using the same methods as those in basic SELEX. Aptamer B3 showed the highest binding affinity for $S$. aureus, followed by B15, B6, and B7. Aptamer B7 demonstrated a low specificity for E. coli (non-target bacteria), but the remaining aptamers showed a similar low detection level for this organism (Figure S2). According to the binding affinity and selectivity as determined by FACS analysis, aptamer A14, B3, and B6 were selected as showing specific binding to $S$. aureus. Although the exclusivity of selected aptamers was low with common Gram-negative foodborne pathogens, there might be higher cross reactivity with Gram-positive bacteria because of the similarities in cell structure. 
When we compared the selective affinity of aptamers between the basic and modified SELEX procedures, the binding affinities of group A were superior to those of group B, but their selectivity was less. Although the SELEX combined with FACS analysis would be expected higher binding affinity to more than that obtained with basic SELEX, it was not agreed selectivity and specificity of aptamers in this study. This trend is likely due to the random selectivity of the aptamer [16]. SELEX involves selection from a random DNA library [24]; in our case, the specific affinities of the DNA library could be increased by FACS, but the binding affinities would be decreased through the same process. In order to compare the efficacy between basic and modified SELEX, we then analyzed the dissociation constants of the aptamers.

\subsection{Dissociation Constants for Aptamers}

Prior to selection of the leading aptamer candidate, the percent binding efficiency of the aptamer for binding to $10^{8} \mathrm{~S}$. aureus cells was evaluated. Aptamer A14 showed the highest binding rate $(50.18 \%$ cells) at an aptamer concentration of $10 \mathrm{nM}$, but other aptamers showed a lower binding rate (Figure 3).

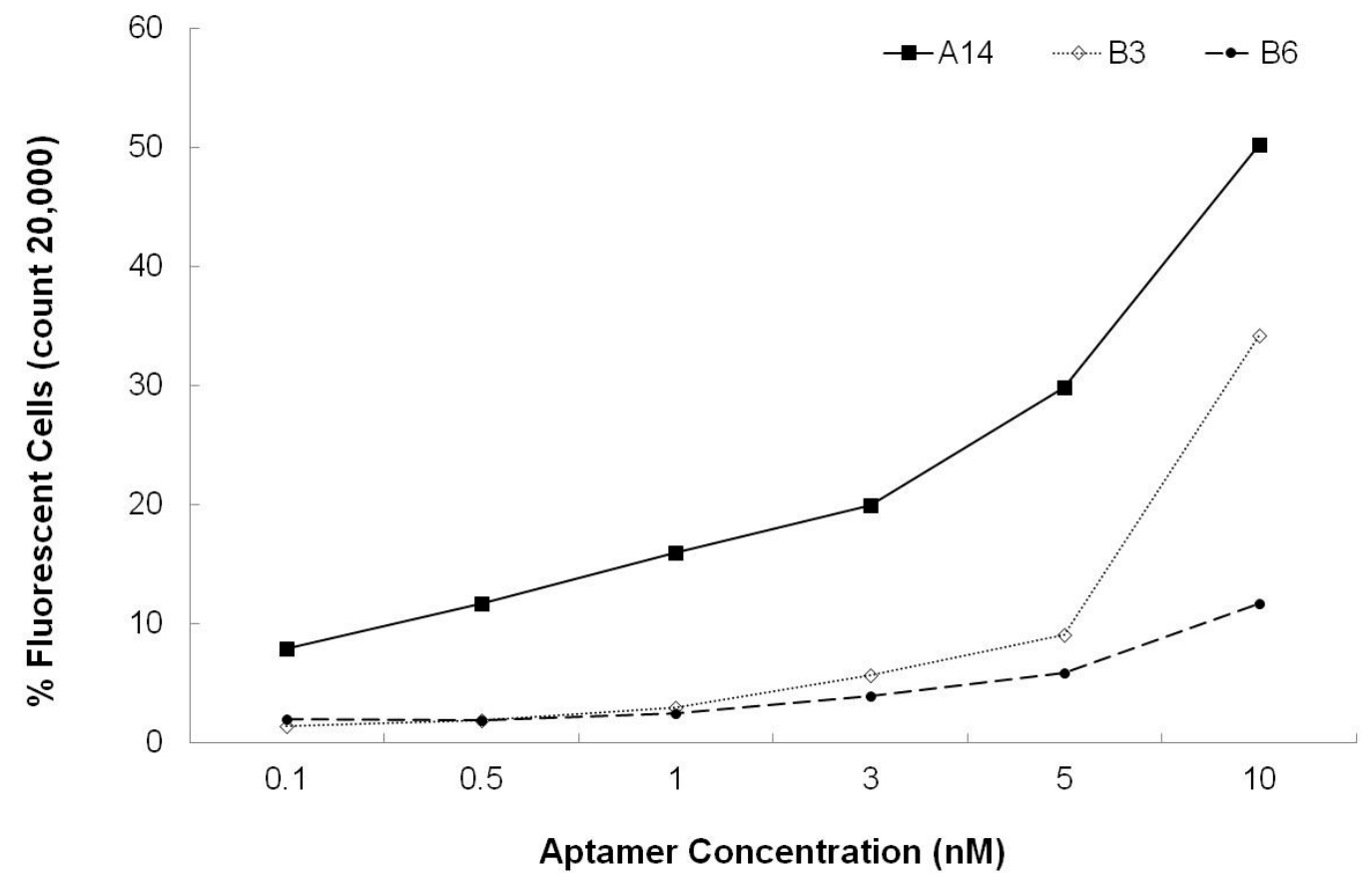

Figure 3. Characterization of aptamers A14, B3, and B6 binding.

In this study, aptamer A14 demonstrated the highest binding affinity, and its equilibrium dissociation constant $(\mathrm{Kd})$ was $3.49 \pm 1.43 \mathrm{nM}$ (Figure 4). These results showed that the binding affinity of aptamer A14 was similar to those of established aptamers specific to $S$. aureus (Kd of SA17-GNPs and SA61-GNPs: $3.03 \mathrm{nM}$ and $9.9 \mathrm{nM}$, respectively) in another study [25]. Compare to Kd values of other developed aptamers for Listeria spp (Kd of LM12-6 and LM6-116: 106.4 and $74.4 \mathrm{nM}$, respectively) [26] and Salmonella typhiamurium (Kd of S8-7: $1.73 \mu \mathrm{M}$ ) [16] aptamer A14 has relatively strong binding affinity. 


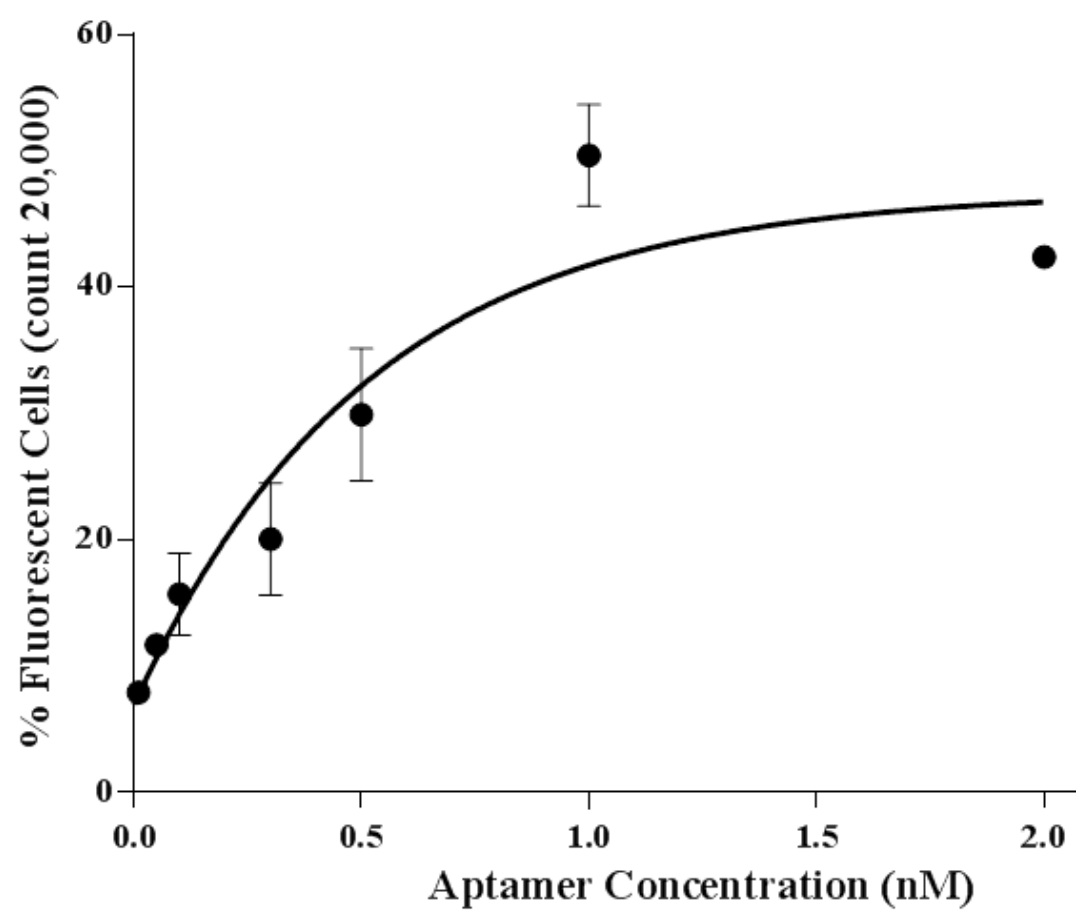

Figure 4. Binding affinity analysis of aptamer A14.

To further investigate aptamer A14, we analyzed its secondary structures. The predicted secondary structure indicates that aptamer A14 could form 2 stem-loop branches and a larger central loop (Figure 5).

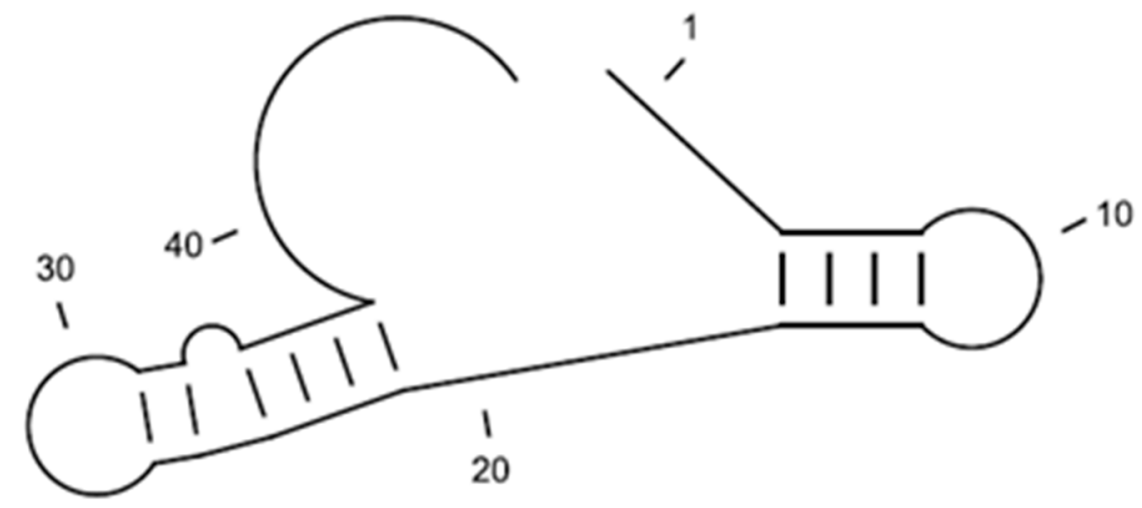

Figure 5. Predicted secondary structure of aptamer A14.

In our previous whole-cell SELEX, $S$. typhimurium was used as target cell [21]. In $S$. aureus cell selection, we expanded the whole-cell SELEX strategy to a cell sorting process. Additionally, combination of separation of binding and cell sorting processes is more effective for improving the specificity of the aptamers than traditional SELEX approaches. The general SELEX process, combined with in vitro selection and amplification, is a technique that can be used for screening very large combinatorial libraries. This selection is divided by positive selection of target cells as well as negative selection. In some studies of aptamer-specific food pathogens, the total number of SELEX rounds were less than or equal to 10. These rounds were composed of SELEX and counter-SELEX, as follows: 3rd SELEX and 4th counter-SELEX against $S$. aureus, 10th SELEX and 2nd counter-SELEX against 
C. jejuni [27], 10th SELEX and 3rd counter-SELEX against $S$. enteritidis [28], 7th-10th SELEX and 2th-6th counter SELEX against $S$. typhimurium [16,21,29,30], and only the 8th SELEX against Lactobacillus acidophilus [22]. These selection processes were designed and performed to improve the specificity and selectivity for target bacteria. In these experiments, cell sorting was performed after the SELEX process. We compared the efficacy of binding of aptamers developed by the basic and modified SELEX process in this study. Our study is the first report that compares the aptamer-binding affinity between basic and modified whole-cell SELEX procedures, aimed at improving selectivity and specificity of DNA aptamers specific for $S$. aureus. This approach presents some hints for designing and optimizing the developing whole-cell SELEX.

Recently, aptamers specific to foodborne pathogens have been developed using whole-cell SELEX [11,14,15,19,21,22,27,28,30]. A total of 18 manuscripts related to the use of aptamers for the identification of $S$. aureus were found in PubMed upon searching the keywords " $S$. aureus" and "aptamer". In these manuscripts, aptamers targeting S. aureus cells were selected and identified, by means of whole-cell SELEX with counter-SELEX [17,25]. Among these manuscripts, no one selected and identified aptamers specific for $S$. aureus cells using FACS sorting. Our study is the first report comparing two kinds of FACS sorting based SELEX approaches to improve the selectivity and specificity of DNA aptamers specific for $S$. aureus. This approach presents an efficient manner for developing aptamers using whole-cell SELEX.

According to reports on the development of aptamers specific for bacteria, whole-cell SELEX has some advantages. First, the aptamers selected by whole-cell SELEX may have affinity for live cells over inactivated cells. Second, whole-cell SELEX could improve the binding affinity of aptamers by combining it with FACS analysis [16].

\section{Conclusions/Outlook}

FACS analysis has been used to isolate DNA bound to target cells and to remove unbound DNAs in whole-cell SELEX. Some studies have suggested that FACS analysis could be implemented in the whole-cell SELEX for perfect separation of bound from unbound sequences [18,31], also which is an important part of SELEX [21]. Considering these characteristics, we suggested two kinds of whole-cell SELEX procedures (Figure 1a,b). We assumed that the modified whole-cell SELEX was superior to the basic model, because the modified model has three FACS analysis steps. However, the binding affinity of aptamers obtained using basic whole-cell SELEX was better in the case of $S$. aureus. Given this result, we suggest that the basic whole-cell SELEX may be suitable for the development of aptamers specific to $S$. aureus. However, the limitation of this study was that it was performed using a single DNA library pool. Although we used a DNA library with a diversity of $10^{14}-10^{16}$, this is a common way to develop aptamers specific to pathogenic bacteria $[16,18,19,21,32]$. The hallmark of the SELEX process is random selection from a pool. Therefore, further study would be needed to perform whole-cell SELEX in larger pools. In summary, our study has suggested the suitability of basic whole-cell SELEX for the development of aptamers specific to $S$. aureus. Moreover, aptamer A14 could be a potential ligand for capturing S. aureus, and may be useful in development of sensitive methodology for bacterial detection. 


\section{Acknowledgments}

This work was carried out with the support of "Cooperative Research Program for Agricultural Science \& Technology Development (Project title: Development of a bioprobe for detection of Staphylococcal enterotoxin, Project No.: PJ00998701)” Rural Development Administration, Korea.

\section{Author Contributions}

Jihea Moon: Performed experiment, wrote the paper: Giyoung Kim; Designed the experiment, wrote the paper: Saet Byeol Park; Performed experiment: Jongguk Lim and Changyeun Mo; Contributed reagents/materials/analysis tools.

\section{Conflicts of Interest}

The authors declare no conflict of interest.

\section{References}

1. Loir, Y.L.; Baron, F.; Gautier, M. Staphylococcus aureus and food poisoning. Gen. Mol. Res. 2003, 2, 63-76.

2. Lowy, F.D. Staphylococcus aureus infections. New Engl. J. Med. 1998, 8, 520-532.

3. Argudín, M.Á.; Mendoza, M.C.; Rodicio, M.R. Food poisoning and Staphylococcus aureus enterotoxins. Toxins 2010, 2, 1751-1773.

4. Han, S.R.; Lee, S.W. In vitro selection of RNA aptamer specific to Staphylococcus aureus. Ann. Microbiol. 2014, 64. 883-885.

5. Food Poisoning Statistics in Korea; Ministry of Food and Drug Safety in Korea: Chungcheongbuk-do, Korea. Available online: http://www.mfds.go.kr (accessed on 1 July 2014).

6. Foodborne Pathogenic Microorganisms and Natural Toxins: Bad Bug Book; US Food and Drug Administration: Silver Spring, MD, USA. Available online: http://www.fda.gov (accessed on 1 July 2014).

7. Duan, N.; Wu, S.; Zhu, C.; Ma, X.; Wang, Z.; Yu, Y.; Jiang, Y. Dual-color upconversion fluorescence and aptamer-functionalized magnetic nanoparticles-based bioassay for the simultaneous detection of Salmonella typhimurium and Staphylococcus aureus. Anal. Chim. Acta 2012, 723, 1-6.

8. Kim, G.; Moon, J.H.; Hahm, B.K.; Morgan, M.; Bhunia, A.; Om, A.S. Rapid detection of Salmonella enteritidis in pork samples with impedimetric biosensor: Effect of electrode spacing on sensitivity. Food Sci. Biotechnol. 2009, 18, 89-94.

9. Kim, G.; Moon, J.H.; Morgan, M. Multivariate data analysis of impedimetric biosensor responses from Salmonella typhimurium. Anal. Methods 2013, 5, 4074-4080.

10. Arora, P; Sindhu, A.; Kaur, H.; Dilbaghi, N.; Chauhury, A. An overview of transducers as platform for the rapid detection of foodborne pathogens. Appl. Microbiol. Biotechnol. 2013, 97, 1829-1840. 
11. Vo-Dinh, T.; Cullum, B. Biosensors and biochips: Advances in biological and medical diagnostics. Fresenius J. Anal. Chem. 2000, 366, 540-551.

12. Ohuchi, S. Cell-SELEX technology. Biores. Open Access 2012, 1, 265-272.

13. Jayasena, S.D. Aptamers: An emerging class of molecules that rival antibodies in diagnostics. Clin. Chem. 1999, 45, 1628-1650.

14. Medley, C.D.; Bamrungsap, S.; Tan, W.; Smith, J.E. Aptamer-conjugated nanoparticles for cancer cell detection. Anal. Chem. 2011, 83, 727-734.

15. Torres-Chavolla, E.E.; Alocilja, E.C. Aptasensors for detection of microbial and viral pathogens. Biosens. Bioelectronics 2009, 24, 3175-3182.

16. Dwivedi, H.P.; Smiley, R.D.; Jaykus, L.A. Selection of DNA aptamers for capture and detection of Salmonella typhimurium using a whole-cell SELEX approach in conjunction with cell sorting. Appl. Microbiol. Biotechnol. 2013, 97, 3677-3686.

17. Cao, X.; Li, S.; Chen, L.; Ding, H.; Xu, H.; Huang, Y.; Li, J.; Liu, N.; Cao, W.; Zhu, Y.; et al. Combining use of a panel of ssDNA aptamers in the detection of Staphylococcus aureus. Nucleic Acids Res. 2009, 37, 4621-4628.

18. Mayer, G.; Ahmed, M.S.L.; Dolf, A.; Endl, E.; Knolle, P.A.; Fumulok, M. Fluorescence-activated cell sorting for aptamer SELEX with cell mixtures. Nat. Protoc. 2010, 5, 1993-2004.

19. Sefah, K.; Shangguan, D.; Xiong, X.; O’Donoghue, M.B.; Tan, W. Development of DNA aptamers using cell-SELEX. Nat. Protoc. 2010, 5, 1169-1185.

20. Cibiel, A.; Dupont, D.M.; Ducongé, F. Methods to identify aptamers against cell surface biomarkers. Pharmaceuticals 2011, 4, 1216-1235.

21. Moon, J.; Kim, G.; Lee, S.; Park, S. Identification of Salmonella typhimurium-specific DNA aptamers developed using whole-cell SELEX and FACS analysis. J. Microbiol. Methods 2013, 95, 162-166.

22. Hamula, C.L.A.; Zhang, H.; Guan, L.L.; Li, X.F.; Le, X.C. Selection of aptamers against live bacterial cells. Anal. Chem. 2008, 80, 7812-7819.

23. Houston, P.; Kodadek, T. Spectrophotometric assay for enzyme-mediated unwinding of double-stranded DNA. Proc. Natl. Acad. Sci. USA 1994, 91, 5471-5474.

24. Stoltenburg, R.; Reinemann, C.; Strehlitz, B. SELEX-A (r)evolutionary method to generate high-affinity nucleic acid ligands. Biomol. Eng. 2007, 24, 381-403.

25. Chang, Y.C.; Yang, C.Y.; Sun, R.L.; Cheng, Y.F.; Kao, W.C.; Yang, P.C. Rapid single cell detection of Staphylococcus aureus by aptamer-conjugated gold nanoparticles. Sci. Rep. 2013, doi:10.1038/srep01863.

26. Suh, S.H.; Dwivedi, H.P.; Choi, S.J.; Jaykus, L.A. Selection and characterization of DNA aptamers specific for Listeria species. Anal. Biochem. 2014, 459, 39-45.

27. Dwivedi, H.P.; Smiley, R.D.; Jaykus, L.A. Selection and characterization of DNA aptamers with binding selectivity to Campylobacter jejuni using whole-cell SELEX. Appl. Microbiol. Biotechnol. 2010, 87, 2323-2334.

28. Hyeon, J.Y.; Chon, J.W.; Choi, I.S.; Park, C.; Kim, D.E.; Seo, K.H. Development of RNA aptamers for detection of Salmonella Enteritidis. J. Microbiol. Methods 2012, 87, 79-82. 
29. Joshi, R.; Janagama, H.; Dwivedi, H.P.; Kumar, T.M.A.S.; Jaykus, L.A. Selection, characterization, and application of DNA aptamers for the capture and detection of Salmonella enterica serovars. Mol. Cell. Probes 2009, 23, 20-28.

30. Lee, Y.J.; Han, S.Y.; Maeng, J.S.; Cho, Y.J.; Lee, S.W. In vitro selection of Escherichia coli O157:H7-specific RNA aptamer. Biochem. Bioph. Res. Commun. 2011, 417, 414-420.

31. Meyer, M.; Scheper, T.; Walter, J.G. Aptamers: Versatile probes for flow cytometry. Appl. Microbiol. Biotechnol. 2013, 97, 7097-7109.

32. Meyer, S.; Maufort, J.P.; Nie, J.; Stewart, R.; Mclntosh, B.E.; Conti, L.R.; Ahmad, K.M.; Soh, H.T.; Thomson, J.A. Development of an efficient targeted cell-SELEX procedure for DNA aptamer reagents. PLOS ONE 2013, 8, e71798.

(C) 2015 by the authors; licensee MDPI, Basel, Switzerland. This article is an open access article distributed under the terms and conditions of the Creative Commons Attribution license (http://creativecommons.org/licenses/by/4.0/). 